\title{
Larvicidal activity of indigenous plant extracts on the rural malarial vector, Anopheles culicifacies Giles. (Diptera: Culicidae)
}

\author{
K. Kovendan, ${ }^{1}$ P. Mahesh Kumar, ${ }^{1}$ J. Subramaniam, ${ }^{1}$ K. Murugan, ${ }^{1}$ S. John William² \\ 'Division of Entomology, Department of Zoology, School of Life Sciences, Bharathiar University, \\ Coimbatore; ${ }^{2}$ P.G. Research \& Department of Advanced Zoology and Biotechnology, Loyola College, \\ Nungambakkam, Chennai, Tamil Nadu, India
}

\begin{abstract}
Vector control is one of the most important components in combating vector-borne diseases throughout the world. Application of insecticides is a widely known and popular vector control strategy. The objective of the present study was to evaluate the larvicidal activity of the hexane, diethyl ether, ethyl acetate and acetone extracts of Abutilon indicum, Hyptis suaveolens and Leucas aspera against third-stage larvae of Anopheles culicifiacies. The results clearly suggest that all three selected plant extracts exhibited moderate larvicidal activity after 24 , 48 and $72 \mathrm{~h}$ at 250, 500, 750 and $1000 \mathrm{ppm}$; the lethal concentrations (LC) at $50 \%$ and $90 \%$ of $A$. indicum, $H$. suaveolens against third instar larvae at 24, 48 and $72 \mathrm{~h}$ (hexane, diethyl ether, ethyl acetate and acetone) were as follows: $A$. indicum, $\mathrm{LC}_{50}=1031.65,949.18,833.58$ and
\end{abstract}

Correspondence: Kalimuthu Kovendan, Division of Entomology, Department of Zoology, School of Life Sciences, Bharathiar University, Coimbatore - 641 046, India.

Tel.: +91.9962447932 - Fax: +91.422 .2422387 .

E-mail: gokulsuryah@gmail.com

Key words: Abutilon indicum, Hyptis suaveolens, Leucas aspera, Anopheles culicifiacies, larvicidal activity.

Funding: the authors are thankful to Science Engineering Research Board (SERB), Department of Science and Technology (DST), Govt. of India, New Delhi (SR/FT/LS-156/2012) for providing financial support for the present work.

Acknowledgements: the authors are grateful to Mr. M. Munirathnam, (ICMR, Madurai), Taxonomy and Field Station in Coimbatore, Tamil Nadu, for helping in mosquito collection and identifying mosquito species of samples for the experiment work.

Received for publication: 13 June 2013.

Revision received: 18 April 2014.

Accepted for publication: 12 May 2014.

(C) Copyright K. Kovendan et al., 2014

Licensee PAGEPress, Italy

Journal of Entomological and Acarological Research 2014; $46: 1747$

doi:10.4081/jear.2014.1747

This article is distributed under the terms of the Creative Commons Attribution Noncommercial License (by-nc 3.0) which permits any noncommercial use, distribution, and reproduction in any medium, provided the original author(s) and source are credited.
$673.68 \mathrm{ppm} ; \mathrm{LC}_{90}=2215.87,2234.39,2152.97$ and $2455.10 \mathrm{ppm} ; H$. suaveolens, $\mathrm{LC}_{50}=423.00,347.50,236.58$ and $217.24 \mathrm{ppm}$; $\mathrm{LC}_{90}=1431.91,1292.15,1138.49$ and $1049.27 \mathrm{ppm}$ and $L$. aspera, $\mathrm{LC}_{50}=559.77,401.56,299.71$ and $263.01 \mathrm{ppm} ; \mathrm{LC}_{90}=1400.80,1549.31$, 1157.96 and $1108.72 \mathrm{ppm}$ at $24 \mathrm{~h}$, respectively. Overall, the highest larvicidal activity was observed with $H$. suaveolens extract followed by $L$. aspera and $A$. indicum at various concentrations at 48 and $72 \mathrm{~h}$, respectively. The objective of this investigation was an attempt to search for a user- and eco-friendly vector control agent. The study proved that the selected plant leaf extracts could serve as potent larvicidal agents against $A$. culicifacies in vector control programs.

\section{Introduction}

Malaria is a disease that inflicts a serious negative impact on public health and socio-economic development in resource-limited settings of the world. Malaria directly or indirectly affects the health and wealth of individuals as well as nations. Indeed, malaria is identified both as a disease associated with and a cause of poverty (Karunamoorthi, 2012). Currently, malaria control is hampered by many operational and technical problems. However, the development of insecticidal resistance in malaria vectors to existing conventional insecticides has made malaria vector control more challenging (Sharma \& Saxena, 1996). Anopheles stephensi and Anopheles culicifacies are the two primary malarial vectors in India. In India, there are five sibling species reported, named A, $\mathrm{B}, \mathrm{C}, \mathrm{D}$, and E; however, species A, C, D, and E are considered to be major vectors, while B is a minor vector (Subbarao et al., 1999). Amerasinghe et al. (1999) reported that $A$. culicifacies is the main vector and A. subpictus is a significant secondary malarial vector in Sri Lanka. In India, A. culicifacies is a primary vector in rural as well as periurban areas, which constitutes nearly $65 \%$ of all malaria cases. At the moment, dichlorodiphenyltrichloroethane (DDT) (organochlorine), malathion (organophosphorus) and $\delta$-methrin, cyfluthrin, $\alpha$-cypermethrin and $\lambda$-cyhalothrin (synthetic pyrethroids) are the most commonly applied insecticides for vector control in the public health sector.

Insecticide susceptibility of $A$. culicifacies to DDT, malathion and methrin was evaluated in 2009 in several districts of India by adopting the WHO standard protocol for adult susceptibility (WHO, 1998). It was found that $A$. culicifacies has developed resistance to all insecticides tested (Mishra et al., 2012). Currently, malaria control largely relies on a limited arsenal of materials; viz., artemisinin derivative drugs and pyrethroids. However, these products could also become ineffective due to the continuing evolution of resistance development. In this context, a new innovative user- and eco-friendly alternative vector control strategy is mandatory. A search for powerful contextual communitybased vector control interventions is therefore warranted. 
Abutilon indicum (Malvaceae), which is commonly known as Thuthi (the vernacular name in Tamil), is distributed throughout the driest areas in India (Chopra et al., 1992). It is well known and reputable in the Tamil traditional medicinal system called Siddha as a phytotherapeutic agent against various illnesses such as jaundice, piles, ulcers and leprosy (Yoganarasimhan, 2000). It is also reported to possess effective analgesic activity; a study by Ahmed et al. (2000) indicated that an $80 \%$ ethanol root extract of $A$. indicum had a potential effect against $A$. aegypti and guppy fish (Promsiri et al., 2006). The larvicidal activity of crude hexane, ethyl acetate, petroleum ether, acetone, and methanol extracts of A. indicum, Aegle marmelos, Euphorbia thymifolia, Jatropha gossypifolia and Solanum torvum were evaluated (Rahuman, 2008). Although Hyptis suaveolens is a native plant of tropical America, it is also widespread in tropical Africa, Asia and Australia. It grows under a wide range of soil and climatic conditions, mainly in warm areas of the country.

H. suaveolens is also administered as a traditional medicine for treatment of various ailments, and its essential oil possesses insecticidal and larvicidal properties (Peerzada, 1997; Azevedo et al., 2001). In Nigeria, ethanol extracts of orange peel (Citrus sinensis) and bush tea leaves of H. suaveolens were compared for their toxicity against A. aegypti (Amusan et al., 2005). It has been reported that $H$. suaveolens extract causes notable mortality of $A$. aegypti larvae because the extract also contained insecticidal compounds such as a-tepinoline, a monoterpene that is similar in action to d-limonone, which is present in C. sinensis.

Leucas aspera (Labiatae) is a small herbaceous plant. It is commonly administered as an antipyretic herb in South India. The juice of its leaves is used for psoriasis and swellings as an external application. The plant extract mixed with honey is a good remedy for stomach pain and indigestion. Preliminary chemical examination of $L$. aspera revealed the presence of triterpenoids (Kamar \& Singh, 1994). The whole plant is reported to contain oleanolic acid, ursolic acid, and 3-itosterol (Chaudhury \& Ghosh, 1969). Aerial parts are reported to contain nicotine (Mangathayaru et al., 2006). The flower is reported to contain ten compounds, among them amyl propionate (15.2\%) and isoamyl propionate (14.4\%), which were dominant (Kalachaveedu et al., 2006). L. aspera leaves are used as an insecticide and mosquito repellent in rural areas (Kiritikar and Basu, 1990; Sadhu et al., 2003). The hexane crude extracts of $L$. aspera showed high larvicidal activity against $C$. quinquefasciatus and A. aegypti (Maheswaran et al., 2008; Kovendan et al., 2012b). The aim of the present investigation was to determine the effect of $A$. indicum, $H$. suaveolens and $L$. aspera leaf extracts against third-stage larvae of $A$. culicifacies as a target species.

\section{Materials and methods}

\section{Collection and rearing of mosquitoes}

Larvae of $A$. culicifacies were collected from Kallar village, near Mettupalayam, Tamil Nadu, in different breeding habitats in using an $O$ type brush. The mosquito larvae were fed with pedigree dog biscuits and yeast in a 3:1 ratio. Feeding was continued until the larvae transformed into the pupal stage. The pupae were collected from the culture trays using a dipper and transferred to plastic containers $(12 \times 12 \mathrm{~cm})$ containing $500 \mathrm{~mL}$ of water. The plastic jars were kept in a $90 \times 90 \times 90$ $\mathrm{cm}$ mosquito cage for adult emergence. Mosquito larvae were maintained at $27+2^{\circ} \mathrm{C}, 75-85 \%$ relative humidity, under a photoperiod of 14:10 (light/dark). A 10\% sugar solution was provided for a period of 3 days before blood feeding. The adult female mosquitoes were allowed to feed on the blood of a rabbit (one rabbit per day, exposed on the dorsal side) for 2 days to ensure adequate blood feeding for 5 days. After blood feeding, enamel trays with water from the culture trays were placed in the cage as oviposition substrates.

\section{Collection of plants and preparation of plant extracts}

The selected medicinal plants were collected in and around Maruthamalai hills and Bharathiar University Campus, Coimbatore, Tamil Nadu. The fresh aerial part of $A$. indicum, and fresh leaves of $H$. suaveolens and $L$. aspera were washed thoroughly with tap water and shade dried at room temperature $\left(28 \pm 2^{\circ} \mathrm{C}\right)$ for 5 to 12 days. The airdried materials were powdered separately using a commercial electric blender. From each plant, $300 \mathrm{~g}$ of powdered material was macerated with $1.0 \mathrm{~L}$ of hexane, diethyl ether, ethyl acetate and acetone, sequentially, for a period of $72 \mathrm{~h}$ each and filtered. The yield of the $A$. indicum, $H$. suaveolens and $L$. aspera crude extracts with hexane, diethyl ether, ethyl acetate and acetone, were: A. indicum 8.94, 10.15, 9.36 and 11.55 $\mathrm{g}$ and $H$. suaveolens $8.12,9,66,10.47$, and $9.73 \mathrm{~g}$ and $L$. aspera 11.12 , $8.29,9.34$ and $10.13 \mathrm{~g}$, respectively. The extracts were concentrated at a reduced temperature on a rotary vacuum evaporator and stored at a temperature of $4^{\circ} \mathrm{C}$. One gram of the plant residue was dissolved in 100 $\mathrm{mL}$ of acetone, which was considered as a $1 \%$ stock solution, from which concentrations were prepared ranging from 250, 500, 750 and 1000 ppm, respectively.

\section{Larval toxicity test}

Larvicidal activity was assessed using the procedure of WHO (1996) with slight modifications. A laboratory colony of $A$. culicifacies larvae was used for the larvicidal activity. Twenty-five third-instar larvae of $A$. culicifiacies were kept in 250-mL glass containers, containing $200 \mathrm{~mL}$ of dechlorinated water. Five replicates were set up for each concentration $(250,500,750,1000 \mathrm{ppm})$ and mixed with acetone and Triton- 80 (mixing solution). Larval mortality was assessed at 24, 48 and $72 \mathrm{~h}$. Each experiment was replicated 3 times at room temperature $\left(28 \pm 2^{\circ} \mathrm{C}\right)$ for three different plants. The control mortalities were corrected by using Abbott's formula (Abbott, 1925).

Corrected mortality $=$

Observed mortality in treatment - Observed mortality in control $\times 100$ 100 - Control mortality

Percentage mortality $=\frac{\text { Number of dead larvae }}{\text { Number of larvae introduced }} \times 100$

The lethal concentrations (LC) at 50\% and 90\% were calculated from toxicity data using probit analysis (Finney, 1971).

\section{Statistical analysis}

The average larval mortality data were subjected to probit analysis for calculating $\mathrm{LC}_{50}$ and $\mathrm{LC}_{90}$, and other statistics at a $95 \%$ upper fiducidal limit and lower fiducidal limit, and chi-square values were calculated using SPSS 9.0 version (Statistical software package; StataCorp., College Station, TX, USA). Results at $\mathrm{P}<0.05$ were considered to be statistically significant.

\section{Results}

Preliminary screening is a good means of evaluating the potential larvicidal activity of crude plant extracts, which is often assessed using different solvent extracts. Mortality from the three plants tested is presented in Tables 1-3. At $24 \mathrm{~h}, A$. indicum demonstrated mortality levels in hexane, diethyl ether, ethyl acetate and actone extracts ranging from $22.18,27.32,32.55$ and $41.23 \%$ at $24 \mathrm{~h}$ at $250 \mathrm{ppm}$ to $51.65,56.12,61.55$ and $64.35 \%$ at $1000 \mathrm{ppm}$, respectively (Table 1 ). For H. suaveolens, mortality levels at $24 \mathrm{~h}$ from these extracts ranged from $41.15,46.33,50.60$ and $52.15 \%$ at $250 \mathrm{ppm}$, to $77.18,83.14,88.15$ and $90.77 \%$ at $1000 \mathrm{ppm}$, 
respectively (Table 2). Finally, for $L$. aspera, mortality at $24 \mathrm{~h}$ from these same extracts ranged from $31.12,44.50,46.66$, and $49.15 \%$ at $250 \mathrm{ppm}$, to $74.18,75.27,87.55$ and $89.26 \%$ at $1000 \mathrm{ppm}$, respectively (Table 3). We observed correspondingly higher larval mortality from these extracts at higher concentrations after 48 and $72 \mathrm{~h}$, respectively (Tables 2 and 3).

The $\mathrm{LC}_{50}$ and $\mathrm{LC}_{90}$ values of hexane, diethyl ether, ethyl acetate and acetone extracts of $A$. indicum, $H$. suaveolens and $L$. aspera at 24 were as follows: $A$. indicum, $\mathrm{LC}_{50}=1031.65,949.18,833.58$ and $673.68 \mathrm{ppm}$; and $\mathrm{LC}_{90}=2215.87,2234.39,2152.97$ and $2455.10 \mathrm{ppm}$, respectively. For H. suaveolens, $\mathrm{LC}_{50}=423.00,347.50,236.58$ and $217.24 \mathrm{ppm}$; and $\mathrm{LC}_{90}=1431.91,1292.15,1138.49$ and $1049.27 \mathrm{ppm}$, respectively. Finally, for $L$. aspera, $\mathrm{LC}_{50}=559.77,401.56,299.71$ and $263.01 \mathrm{ppm}$; and $\mathrm{LC}_{90}=1400.80,1549.31,1157.96$ and $1108.72 \mathrm{ppm}$ at $24 \mathrm{~h}$, respectively (Tables 1-3).

\section{Discussion}

A. culicifacies is one of the major malaria vectors in the Indian subcontinent and is generally regarded as intolerant to salinity, preferring to breed in newly-dug freshwater pits, domestic wells and sites used for plantings of coconuts and casurina trees in India (Russell \& Rao, 1942; Sabesan et al., 1986). Ansari et al. (2000) suggested that the peppermint oil (Mentha piperita) showed strong repellent action against adult mosquitoes when applied on human skin. The protection obtained against $A$. annularis, A. culicifacies, and $C$. quinquefasciatus was 100 , 92.3 , and $84.5 \%$, respectively. The present investigation tested three plant leaf extracts in different solvents for their potential larvicidal activity against $A$. culicifacies. The biological activity of the experimental plant extracts varied, which may be due to the presence of various phytochemically active compounds in the plants, including phenolics, terpenoides, flavonoids and alkaloids. These active principles may have jointly or independently influenced or contributed to produce larvicidal effects against $A$. culicifacies.

Sharma \& Ansari (1994) demonstrated the protective effect of Cyperus rotundus against $A$. culicifacies, A. stephensi and $C$. quinquefasciatus, and showed greater protection than neem oil (37.5\%). Sharma et al. (1995), reported that the crude extract of Solanum nigram leaves showed significant larvicidal activity against $A$. culcifacies, $C$. quinquefasciatus and $A$. aegypti at a dose equivalent to the $\mathrm{LC}_{90}$, ranging from 0.18 to $0.21 \%$ (Singh et al., 2002). The present results concur with some of the previous findings of $A$. indicum against the third instar larvae of $A$. culicifacies, with $\mathrm{LC}_{50}$ and $\mathrm{LC}_{90}$ values of hexane, diethyl ether, ethyl acetate and acetone extracts of $\left(\mathrm{LC}_{50}\right)$ 1031.65, 949.18, 833.58 and $673.68 \mathrm{ppm}$, and ( $\left.\mathrm{LC}_{90}\right)$ 2215.87, 2234.39, 2152.97 and $2455.10 \mathrm{ppm}$ at $24 \mathrm{~h}$, respectively.

One study reported that the lethal concentration values of the aqueous extract of roots of $H$. abelmoschus against the larvae of $A$. culicifacies, $A$. stephensi, and $C$. quinquefasciatus were $52.3,52.6$, and $43.8 \mathrm{ppm}$, respectively (Dua et al., 2006). The $\mathrm{LC}_{50}$ and $\mathrm{LC}_{90}$ values of $S$. indicus, $C$. collinus and $M$. koenigii against third-instar larvae at 24,48 and $72 \mathrm{~h}$ (in hexane, chloroform and ethyl acetate extracts) were: for $S$. indicus, $\left(\mathrm{LC}_{50}\right)$ 544.93, 377.86 and $274.79 \mathrm{ppm}$, and ( $\left.\mathrm{LC}_{90}\right)$ 1325.32, 1572.55 and $1081.29 \mathrm{ppm}$ at $24 \mathrm{~h}$; for $C$. collinus, $\left(\mathrm{LC}_{50}\right) 375.34,318.29$ and 226.10 ppm, and ( $\left.\mathrm{LC}_{90}\right)$ 699.65, 1577.62 and $1024.92 \mathrm{ppm}$ at $24 \mathrm{~h}$; and, for $M$. koenigii, ( $\left.\mathrm{LC}_{50}\right)$ 963.53, 924.85 and $857.62 \mathrm{ppm}$, and ( $\left.\mathrm{LC}_{90}\right)$ 1665.12, 1624.68 and $1564.37 \mathrm{ppm}$ at $24 \mathrm{~h}$, respectively (Kovendan et al., 2012a).

Table 1. Larvicidal activity of Abutilon indicum against third instars larvae of Anopheles culicifacies.

\begin{tabular}{|c|c|c|c|c|c|c|c|c|c|}
\hline \multirow[t]{2}{*}{$\begin{array}{l}\text { Exposure } \\
\text { (time) }\end{array}$} & \multirow[t]{2}{*}{ Solvents } & \multicolumn{4}{|c|}{$\begin{array}{l}\text { Percentage larval mortality } \pm \text { SD } \\
\text { Concentration of } A \text {. indicum (ppm) }\end{array}$} & \multirow[t]{2}{*}{$\begin{array}{c}\mathrm{LC}_{50} \\
\left(\mathrm{LC}_{90}\right)\end{array}$} & \multicolumn{2}{|c|}{$\begin{array}{l}95 \% \text { confidence limit } \\
\text { LFL UFL }\end{array}$} & \multirow[t]{2}{*}{$\begin{array}{c}x^{2} \\
(d f=4)\end{array}$} \\
\hline & & 250 & 500 & 750 & 1000 & & $\mathrm{LC}_{50}\left(\mathrm{LC}_{90}\right)$ & $\mathrm{LC}_{50}\left(\mathrm{LC}_{90}\right)$ & \\
\hline \multirow{4}{*}{$24 \mathrm{~h}$} & Hexane & $22.18 \pm 0.86$ & $26.31 \pm 1.12$ & $34.56 \pm 0.98$ & $51.65 \pm 1.50$ & $\begin{array}{c}1031.65 \\
(2215.87)\end{array}$ & $\begin{array}{c}877.34 \\
(1733.59)\end{array}$ & $\begin{array}{c}1360.29 \\
(3414.23)\end{array}$ & $1.39^{\mathrm{a}}$ \\
\hline & Diethyl ether & $27.32 \pm 0.42$ & $30.40 \pm 0.65$ & $37.19 \pm 1.32$ & $56.12 \pm 1.25$ & $\begin{array}{c}949.18 \\
(2234.39)\end{array}$ & $\begin{array}{c}802.18 \\
(1722.60)\end{array}$ & $\begin{array}{c}1260.00 \\
(3594.44)\end{array}$ & $2.41^{\mathrm{a}}$ \\
\hline & Ethyl acetate & $32.55 \pm 1.23$ & $34.12 \pm 1.45$ & $40.66 \pm 0.44$ & $61.55 \pm 1.18$ & $\begin{array}{c}833.58 \\
(2152.97)\end{array}$ & $\begin{array}{c}700.82 \\
(1659.17)\end{array}$ & $\begin{array}{c}1076.37 \\
(3484.70)\end{array}$ & $3.78^{\mathrm{a}}$ \\
\hline & Acetone & $41.23 \pm 1.65$ & $43.65 \pm 0.68$ & $45.22 \pm 1.36$ & $64.35 \pm 1.33$ & $\begin{array}{c}673.68 \\
(2455.10)\end{array}$ & $\begin{array}{c}479.50 \\
(1742.72)\end{array}$ & $\begin{array}{c}928.05 \\
(5435.70)\end{array}$ & $3.52^{\mathrm{a}}$ \\
\hline \multirow{4}{*}{$48 \mathrm{~h}$} & Hexane & $30.63 \pm 0.54$ & $38.50 \pm 1.69$ & $55.60 \pm 1.74$ & $68.92 \pm 1.95$ & $\begin{array}{c}655.69 \\
(1586.80)\end{array}$ & $\begin{array}{c}561.41 \\
(1333.98)\end{array}$ & $\begin{array}{c}757.04 \\
(2079.68)\end{array}$ & $0.56^{\mathrm{a}}$ \\
\hline & Diethyl ether & $34.12 \pm 1.55$ & $42.26 \pm 0.72$ & $58.77 \pm 0.95$ & $71.23 \pm 1.27$ & $\begin{array}{c}592.82 \\
(1556.39)\end{array}$ & $\begin{array}{c}487.55 \\
(1303.21)\end{array}$ & $\begin{array}{c}690.49 \\
(2061.01)\end{array}$ & $0.48^{\mathrm{a}}$ \\
\hline & Ethyl acetate & $44.35 \pm 1.21$ & $49.22 \pm 1.56$ & $61.10 \pm 1.22$ & $74.88 \pm 1.48$ & $\begin{array}{c}444.90 \\
(1625.26)\end{array}$ & $\begin{array}{c}255.91 \\
(1310.21)\end{array}$ & $\begin{array}{c}562.52 \\
(2373.37)\end{array}$ & $1.10^{\mathrm{a}}$ \\
\hline & Acetone & $50.56 \pm 1.35$ & $63.91 \pm 1.75$ & $71.25 \pm 1.66$ & $85.45 \pm 1.30$ & $\begin{array}{c}274.54 \\
(1207.15)\end{array}$ & $\begin{array}{c}69.32 \\
(1026.23)\end{array}$ & $\begin{array}{c}392.03 \\
(1561.63)\end{array}$ & $0.76^{\mathrm{a}}$ \\
\hline \multirow{4}{*}{$72 \mathrm{~h}$} & Hexane & $48.61 \pm 1.52$ & $65.22 \pm 1.88$ & $84.14 \pm 1.47$ & $91.35 \pm 1.32$ & $\begin{array}{c}273.83 \\
(935.20)\end{array}$ & $\begin{array}{c}140.65 \\
(831.15)\end{array}$ & $\begin{array}{c}362.35 \\
(1100.14)\end{array}$ & $0.46^{\mathrm{a}}$ \\
\hline & Diethyl ether & $51.70 \pm 0.80$ & $70.50 \pm 1.95$ & $91.36 \pm 1.93$ & $96.50 \pm 1.40$ & $\begin{array}{c}245.95 \\
(764.39)\end{array}$ & $\begin{array}{c}136.20 \\
(688.68)\end{array}$ & $\begin{array}{c}321.82 \\
(873.81)\end{array}$ & $0.99^{\mathrm{a}}$ \\
\hline & Ethyl acetate & $58.44 \pm 1.65$ & $79.20 \pm 1.20$ & $93.18 \pm 1.65$ & $98.42 \pm 1.91$ & $\begin{array}{c}230.45 \\
(676.51)\end{array}$ & $\begin{array}{c}130.83 \\
(611.13)\end{array}$ & $\begin{array}{c}299.81 \\
(767.98)\end{array}$ & $0.14^{\mathrm{a}}$ \\
\hline & Acetone & $61.12 \pm 1.40$ & $80.10 \pm 1.33$ & $96.54 \pm 1.21$ & $100.00 \pm 0.00$ & $\begin{array}{c}179.87 \\
(594.49)\end{array}$ & $\begin{array}{c}66.42 \\
(532.79)\end{array}$ & $\begin{array}{c}253.45 \\
(681.76)\end{array}$ & $1.95^{\mathrm{a}}$ \\
\hline
\end{tabular}

Control, nil mortality; SD, standard deviation; $\mathrm{LC}_{50}$, $\mathrm{LC}_{90}$, lethal concentration at $50 \%$ and $90 \%$; $\mathrm{LFL}$, lower fiducidal limit; UFL, upper fiducidal limit; $x^{2}$, Chi-square value; $d f$, degrees of freedom. Mean values of five replicates. ${ }^{\text {SSignificant at } \mathrm{P}<0.05 \text { level. }}$ 
Table 2. Larvicidal activity of Hyptis suaveolens against the third instars larvae of Anopheles culicifacies.

\begin{tabular}{|c|c|c|c|c|c|c|c|c|c|}
\hline $\begin{array}{l}\text { Exposure } \\
\text { (time) }\end{array}$ & Solvents & \multicolumn{4}{|c|}{$\begin{array}{l}\text { Percentage larval mortality } \pm \text { SD } \\
\text { Concentration of } \boldsymbol{H} \text {. suaveolens }(\mathrm{ppm})\end{array}$} & $\begin{array}{l}\mathrm{LC}_{50} \\
\left(\mathrm{LC}_{90}\right)\end{array}$ & \multicolumn{2}{|c|}{$\begin{array}{l}\text { 95\% confidence limit } \\
\text { LFL } \\
\text { LC }_{50}\left(\mathrm{LC}_{90}\right) \quad \mathrm{LC}_{50}\left(\mathrm{LC}_{90}\right)\end{array}$} & $\begin{array}{c}x^{2} \\
(d f=4)\end{array}$ \\
\hline \multirow{4}{*}{$24 \mathrm{~h}$} & Hexane & $41.15 \pm 0.94$ & $54.59 \pm 1.23$ & $65.20 \pm 1.13$ & $77.18 \pm 1.19$ & $\begin{array}{c}423.00 \\
(1431.91)\end{array}$ & $\begin{array}{c}262.74 \\
(1195.44)\end{array}$ & $\begin{array}{c}527.13 \\
(1920.40)\end{array}$ & $0.06^{\mathrm{a}}$ \\
\hline & Diethyl ether & $46.33 \pm 1.89$ & $57.16 \pm 0.88$ & $68.15 \pm 0.92$ & $83.14 \pm 1.93$ & $\begin{array}{c}347.50 \\
(1292.15)\end{array}$ & $\begin{array}{c}171.86 \\
(1094.04)\end{array}$ & $\begin{array}{c}454.55 \\
(1682.95)\end{array}$ & $0.72^{\mathrm{a}}$ \\
\hline & Ethyl acetate & $50.60 \pm 1.51$ & $67.24 \pm 0.85$ & $72.13 \pm 1.80$ & $88.15 \pm 1.37$ & $\begin{array}{c}236.58 \\
(1138.49)\end{array}$ & $\begin{array}{c}22.09 \\
(973.62)\end{array}$ & $\begin{array}{c}357.75 \\
(1454.65)\end{array}$ & $1.83^{\mathrm{a}}$ \\
\hline & Acetone & $52.15 \pm 0.88$ & $69.10 \pm 1.46$ & $74.78 \pm 1.69$ & $90.77 \pm 0.98$ & $\begin{array}{c}217.24 \\
(1049.27)\end{array}$ & $\begin{array}{c}15.04 \\
(907.98)\end{array}$ & $\begin{array}{c}334.37 \\
(1305.85)\end{array}$ & $2.00^{\mathrm{a}}$ \\
\hline \multirow{4}{*}{$48 \mathrm{~h}$} & Hexane & $43.18 \pm 0.56$ & $58.14 \pm 0.84$ & $68.93 \pm 0.92$ & $79.50 \pm 1.25$ & $\begin{array}{c}367.14 \\
(1343.81)\end{array}$ & $\begin{array}{c}191.69 \\
(1130.97)\end{array}$ & $\begin{array}{c}474.69 \\
(1772.94)\end{array}$ & $0.08^{\mathrm{a}}$ \\
\hline & Diethyl ether & $48.50 \pm 1.16$ & $63.20 \pm 0.91$ & $70.18 \pm 0.79$ & $86.00 \pm 1.77$ & $\begin{array}{c}284.56 \\
(1207.01)\end{array}$ & $\begin{array}{c}86.93 \\
(1027.61)\end{array}$ & $\begin{array}{c}399.26 \\
(1555.88)\end{array}$ & $1.19^{\mathrm{a}}$ \\
\hline & Ethyl acetate & $51.65 \pm 1.27$ & $68.15 \pm 1.85$ & $75.21 \pm 1.54$ & $89.14 \pm 0.69$ & $\begin{array}{c}219.38 \\
(1081.48)\end{array}$ & $\begin{array}{c}9.19 \\
(931.45)\end{array}$ & $\begin{array}{c}339.44 \\
(1360.06)\end{array}$ & $1.00^{\mathrm{a}}$ \\
\hline & Acetone & $54.01 \pm 0.85$ & $70.36 \pm 0.78$ & $79.36 \pm 1.10$ & $92.56 \pm 0.75$ & $\begin{array}{c}196.53 \\
(962.17)\end{array}$ & $\begin{array}{c}4.99 \\
(841.05) \\
\end{array}$ & $\begin{array}{c}309.95 \\
(1170.61)\end{array}$ & $0.91^{\mathrm{a}}$ \\
\hline \multirow{4}{*}{$72 \mathrm{~h}$} & Hexane & $47.10 \pm 1.27$ & $65.48 \pm 1.35$ & $77.23 \pm 1.16$ & $84.78 \pm 1.68$ & $\begin{array}{c}268.78 \\
(1134.84)\end{array}$ & $\begin{array}{c}80.81 \\
(977.06)\end{array}$ & $\begin{array}{c}380.42 \\
(1426.91)\end{array}$ & $0.51^{\mathrm{a}}$ \\
\hline & Diethyl ether & $57.03 \pm 0.78$ & $79.85 \pm 1.18$ & $85.55 \pm 0.77$ & $98.32 \pm 0.88$ & $\begin{array}{c}164.33 \\
(751.50)\end{array}$ & $\begin{array}{c}7.30 \\
(668.80)\end{array}$ & $\begin{array}{c}262.41 \\
(875.67)\end{array}$ & $3.56^{\mathrm{a}}$ \\
\hline & Ethyl acetate & $70.01 \pm 0.96$ & $97.36 \pm 0.89$ & $100.0 \pm 0.00$ & $100.0 \pm 0.00$ & $\begin{array}{c}158.80 \\
(382.51)\end{array}$ & $\begin{array}{c}56.706 \\
(339.78)\end{array}$ & $\begin{array}{c}211.84 \\
(452.69)\end{array}$ & $0.04^{\mathrm{a}}$ \\
\hline & Acetone & $75.13 \pm 0.70$ & $98.56 \pm 0.00$ & $100.00 \pm 0.00$ & $100.00 \pm 0.00$ & $\begin{array}{c}138.30 \\
(349.48)\end{array}$ & $\begin{array}{c}6.89 \\
(307.90)\end{array}$ & $\begin{array}{c}195.95 \\
(421.39)\end{array}$ & $0.01^{\mathrm{a}}$ \\
\hline
\end{tabular}

Control, nil mortality; SD, standard deviation; $\mathrm{LC}_{50}, \mathrm{LC} 90$, lethal concentration at $50 \%$ and $90 \%$; $\mathrm{LFL}$, lower fiducidal limit; UFL, upper fiducidal limit; $x^{2}$, Chi-square value; $d f$, degrees of freedom. Mean values of five replicates. a Significant at $\mathrm{P}<0.05$ level.

Table 3. Larvicidal activity of Leucas aspera against third instars larvae of Anopheles culicifacies.

\begin{tabular}{|c|c|c|c|c|c|c|c|c|c|}
\hline $\begin{array}{l}\text { Exposure } \\
\text { (time) }\end{array}$ & Solvents & \multicolumn{4}{|c|}{$\begin{array}{l}\text { Percentage larval mortality } \pm \text { SD } \\
\text { Concentration of } L \text {. aspera }(\mathrm{ppm})\end{array}$} & $\begin{array}{c}\mathrm{LC}_{50} \\
\left(\mathrm{LC}_{90}\right)\end{array}$ & \multicolumn{2}{|c|}{$\begin{array}{l}\text { 95\% confidence limit } \\
\text { LFL } \\
\text { LC }_{50}\left(\mathrm{LC}_{90}\right) \quad \mathrm{LC}_{50}\left(\mathrm{LC}_{90}\right)\end{array}$} & $\begin{array}{c}x^{2} \\
(d f=4)\end{array}$ \\
\hline \multirow{4}{*}{$24 \mathrm{~h}$} & Hexane & $31.12 \pm 1.17$ & $47.12 \pm 1.45$ & $62.14 \pm 1.88$ & $74.18 \pm 1.25$ & $\begin{array}{c}559.77 \\
(1400.80)\end{array}$ & $\begin{array}{c}464.21 \\
(1203.49)\end{array}$ & $\begin{array}{c}643.56 \\
(1758.85)\end{array}$ & $0.09^{a}$ \\
\hline & Diethyl ether & $44.50 \pm 1.20$ & $52.43 \pm 1.94$ & $65.36 \pm 1.70$ & $75.27 \pm 1.33$ & $\begin{array}{c}401.56 \\
(1549.31)\end{array}$ & $\begin{array}{c}198.85 \\
(1259.51)\end{array}$ & $\begin{array}{c}520.40 \\
(2219.35)\end{array}$ & $0.22^{\mathrm{a}}$ \\
\hline & Ethyl acetate & $46.66 \pm 1.32$ & $65.16 \pm 1.80$ & $69.52 \pm 1.57$ & $87.55 \pm 0.88$ & $\begin{array}{c}299.71 \\
(1157.96)\end{array}$ & $\begin{array}{c}126.24 \\
(996.97)\end{array}$ & $\begin{array}{c}405.43 \\
(1455.63)\end{array}$ & $2.48^{\mathrm{a}}$ \\
\hline & Acetone & $49.15 \pm 1.50$ & $66.98 \pm 1.57$ & $71.55 \pm 1.36$ & $89.26 \pm 1.44$ & $\begin{array}{c}263.01 \\
(1108.72)\end{array}$ & $\begin{array}{c}77.46 \\
(956.98)\end{array}$ & $\begin{array}{c}373.42 \\
(1387.02)\end{array}$ & $2.56^{\mathrm{a}}$ \\
\hline \multirow{4}{*}{$48 \mathrm{~h}$} & Hexane & $37.92 \pm 0.95$ & $54.35 \pm 1.65$ & $68.12 \pm 1.30$ & $77.10 \pm 1.65$ & $\begin{array}{c}426.33 \\
(1377.58)\end{array}$ & $\begin{array}{c}279.40 \\
(1163.25)\end{array}$ & $\begin{array}{c}524.96 \\
(1800.20)\end{array}$ & $0.14^{\mathrm{a}}$ \\
\hline & Diethyl ether & $45.42 \pm 0.77$ & $61.72 \pm 1.10$ & $73.50 \pm 1.55$ & $82.64 \pm 1.93$ & $\begin{array}{c}312.92 \\
(1223.97)\end{array}$ & $\begin{array}{c}131.45 \\
(1044.38)\end{array}$ & $\begin{array}{c}421.96 \\
(1568.28)\end{array}$ & $0.16^{\mathrm{a}}$ \\
\hline & Ethyl acetate & $48.16 \pm 1.13$ & $69.41 \pm 1.72$ & $76.92 \pm 1.42$ & $89.18 \pm 1.18$ & $\begin{array}{c}253.69 \\
(1038.54)\end{array}$ & $\begin{array}{l}81.55 \\
(906.06)\end{array}$ & $\begin{array}{c}359.15 \\
(1269.33)\end{array}$ & $0.95^{\mathrm{a}}$ \\
\hline & Acetone & $51.75 \pm 1.95$ & $78.56 \pm 1.23$ & $87.30 \pm 1.22$ & $93.48 \pm 1.35$ & $\begin{array}{c}183.68 \\
(824.85)\end{array}$ & $\begin{array}{c}22.88 \\
(732.59)\end{array}$ & $\begin{array}{c}284.62 \\
(967.34)\end{array}$ & $2.14^{\mathrm{a}}$ \\
\hline \multirow{4}{*}{$72 \mathrm{~h}$} & Hexane & $45.60 \pm 1.26$ & $61.54 \pm 0.90$ & $73.81 \pm 1.18$ & $87.36 \pm 1.44$ & $\begin{array}{c}325.57 \\
(1119.70)\end{array}$ & $\begin{array}{c}178.16 \\
(974.76)\end{array}$ & $\begin{array}{c}420.93 \\
(1374.18)\end{array}$ & $0.38^{\mathrm{a}}$ \\
\hline & Diethyl ether & $52.76 \pm 1.17$ & $79.30 \pm 1.29$ & $91.10 \pm 1.94$ & $94.75 \pm 1.20$ & $\begin{array}{c}183.77 \\
(761.60)\end{array}$ & $\begin{array}{c}39.44 \\
(680.09)\end{array}$ & $\begin{array}{c}276.83 \\
(881.77)\end{array}$ & $2.18^{\mathrm{a}}$ \\
\hline & Ethyl acetate & $63.15 \pm 0.92$ & $86.25 \pm 1.12$ & $96.63 \pm 1.61$ & $100.00 \pm 0.00$ & $\begin{array}{c}144.51 \\
(560.83)\end{array}$ & $\begin{array}{c}14.56 \\
(499.23)\end{array}$ & $\begin{array}{c}225.14 \\
(646.87)\end{array}$ & $0.63^{\mathrm{a}}$ \\
\hline & Acetone & $88.80 \pm 1.13$ & $93.24 \pm 1.50$ & $100.00 \pm 0.00$ & $100.00 \pm 0.00$ & $\begin{array}{c}138.24 \\
(478.13)\end{array}$ & $\begin{array}{c}11.798 \\
(423.46)\end{array}$ & $\begin{array}{c}211.30 \\
(557.32)\end{array}$ & $2.54^{\mathrm{a}}$ \\
\hline
\end{tabular}

Control, nil mortality; SD, standard deviation; $\mathrm{LC}_{50}$, LC90, lethal concentration at 50\% and 90\%; LFL, lower fiducidal limit; UFL, upper fiducidal limit; $x^{2}$, Chi-square value; $d f$, degrees of freedom. Mean values of five replicates. aSignificant at $\mathrm{P}<0.05$ level. 
It is known that placing $H$. suaveolens branches or whole plants in and around houses is one of the most effective methods in western Kenya, for repelling the malaria vector Anopheles gambiae Giles (Seyoum et al., 2002). Kovendan et al. (2012b) reported on the effects of hexane, chloroform, ethyl acetate and methanol extracts of $J$. curcas against third instar larvae of $C$. quinquefasciatus, with $\mathrm{LC}_{50}$ values of $230.32,212.85,192.07$ and $113.23 \mathrm{ppm}$, respectively. For H. suaveolens, $\mathrm{LC}_{50}$ values of these extracts were $213.09,217.64,167.59$ and 86.93 ppm, respectively. For $A$. indicum, the $\mathrm{LC}_{50}$ values were $204.18,155.53,166.32$ and $111.58 \mathrm{ppm}$, respectively. Finally, for $L$. aspera, $\mathrm{LC}_{50}$ values were $152.18,118.29,111.43$ and $107.73 \mathrm{ppm}$, respectively. Similarly, our results with $H$. suaveolens against the third-instar larvae of $A$. culicifacies showed $\mathrm{LC}_{50}$ and $\mathrm{LC}_{90}$ values of hexane, diethyl ether, ethyl acetate and acetone extracts of $\left(\mathrm{LC}_{50}\right)$ 423.00, 347.50, 236.58 and $217.24 \mathrm{ppm}$, and ( $\left.\mathrm{LC}_{90}\right)$ 1,431.91, 1292.15, 1138.49 and $1049.27 \mathrm{ppm}$ at $24 \mathrm{~h}$, respectively.

Previous studies have been conducted using a methanol extract of Clerodendron inerme and Acanthus ilicifolius at different concentrations (20, 40, 60, 80 and $100 \mathrm{ppm})$, with $\mathrm{LC}_{50}$ values against $A$. stephen$s i$ first to fourth-instar larvae and pupae of 55.04, 63.33, 73.05, 80.74 and $74.33 \mathrm{ppm}$, and 52.76, 57.76, 63.36, 70.18 and $62.78 \mathrm{ppm}$, respectively. Corresponding $\mathrm{LC}_{90}$ values were $125.50,137.16,153.55,156.93$, $199.20 \mathrm{ppm}$, and 108.30, 115.83, 125.24, 131.28 and $141.03 \mathrm{ppm}$, respectively (Kovendan \& Murugan, 2011). Mahesh Kumar et al. (2012) reported the $\mathrm{LC}_{50}$ and $\mathrm{LC}_{90}$ values of $S$. xanthocarpum against the first to fourth instar larvae and pupae of $C$. quinquefasciatus as 155.29, $198.32,271.12,377.44$, and $448.41 \mathrm{ppm}$, and 687.14, 913.10, 1,011.89, $1,058.85$, and $1,141.65 \mathrm{ppm}$, respectively. In the present results, the hexane, diethyl ether, ethyl acetate and acetone extracts of $L$. aspera against third-instar larvae of $A$. culicifacies had $\mathrm{LC}_{50}$ values of 559.77 , 401.56, 299.71 and $263.01 \mathrm{ppm}$, and $\mathrm{LC}_{90}$ values of 1400.80, 1549.31, 1157.96 and $1108.72 \mathrm{ppm}$ at $24 \mathrm{~h}$, respectively.

\section{Conclusions}

The larvicidal properties of crude extracts of $A$. indicum, $H$. suaveolens and $L$. aspera against $A$. culicifacies were studied under laboratory conditions. The results clearly demonstrated the highest mortality with $H$. suaveolens, followed by $L$. aspera and $A$. indicum. In addition, the study also showed that the solvents used for the extractions have some impact on the level of larval mortality. The highest mortality was seen with acetone extract followed by ethyl acetate, diethyl ether and hexane. This mortality profile demonstrates the extraction properties of different solvents from which the maximum effect was obtained. We conclude that botanicals could be a better alternative than relying on the most hazardous currently used synthetic chemical insecticides and bio-pesticides, and could contribute to a healthier environment. The use of plant-based products could be an ideal eco- and user-friendly vector control strategy for diminishing and eventual elimination of the malaria burden in the near future.

\section{References}

ABBOTT W.S., 1925 - A method of computing the effectiveness of insecticides. - J. Econ. Entomol. 18: 267-269.

AHMED M., AMIN S., ISLAM M., TAKAHASHI M., OKUYAMA E., HOSSAIN C.F., 2000 - Analgesic principle from Abutilon indicum. Pharmazie. 55: 314.

AMERASINGHE P.H., AMERASINGHE F.P., KONRADSEN F., FONSEKA K.T., WIRTZ R.A., 1999 - Malaria vectors in a traditional dry zone village in Sri Lanka. - Am. J. Trop. Med. Hyg. 60: 421-9.
AMUSAN A.A., IDOWU A.B., AROWOLO F.S., 2005 - Comparative toxicity effect of bush tea leaves (Hyptis suaveolens) and orange peel (Citrus sinensis) oil extract on larvae of the yellow fever mosquito Aedes aegypti. - Tanzan. Health. Res. Bull. 7: 174-178.

ANSARI M.A., VASUDEVAN P., TANDON M., RAZDAN R.K., 2000 Larvicidal and mosquito repellent action of peppermint (Mentha piperita) oil. Biores. Technol. - 71: 267-271.

AZEVEDO N.A., CAMPOS L.F.P., FERREIRA H.D., 2001 - Chemical variability in the essential oil of Hyptis suaveolens. - Phytochemistry 57: 733-736.

CHAUDHURY A., GHOSH D., 1969 - Insecticidal plants: chemical examination of leucas aspera. - J. Indian Chem. Soc. 46: 95.

CHOPRA R.N., NAYER S.L., CHOPRA I.C., 1992 - Glossary of Indian Medicinal plants, $3^{\text {rd }}$ ed. Council of Scientific and Industrial Research, New Delhi, pp. 7-246.

DUA V.K., PANDEY A.C., ALAM M.E., DASH A.P., 2006 - Larvicidal activity of Hibiscus abelmoschus Linn. (Malvaceae) against mosquitoes. - J. Am. Mosq. Control. Assoc. 22: 155-157.

FINNEY D.J., 1971 - Probit analysis. Cambridge University Press, London, pp. 68-78.

KALACHAVEEDU M., GHOSH A., RANJAN R., VEDAM VENKAT K., 2006 - Volatile constituents of Leucas aspera (Willd.). - J. Essent. Oil. Res. 18: 104-5.

KAMAR M., SINGH T.P., 1994 - Preliminary chemical examination of some compounds in the different parts of the genus Leucas. Geobios. 21: 31-33.

KARUNAMOORTHI K., 2012 - Global malaria burden: socialomics implications. - J. Socialomics 1: e108.

KITIKAR K.R., BASU B.D., 1990 - Indian medicinal plants. In: BIDTTER E., CAAIUS J.F., MHASKAR K.S. (Eds.). - Periodical Experts Book Company, New Delhi: 2019.

KOVENDAN K., MURUGAN K., 2011 - Effect of medicinal plants on the mosquito vectors from the different agro-climatic regions of Tamil Nadu, India. - Adv. Environ. Biol. 5: 335-344.

KOVENDAN K., ARIVOLI S., MAHESHWARAN R., BASKAR K., VINCENT S., 2012a - Larvicidal efficacy of Sphaeranthus indicus, Cleistanthus collinus and Murraya koenigii leaf extracts against filarial vector, Culex quinquefasciatus Say (Diptera: Culicidae) Parasitol. Res. 111: 1025-1035.

KOVENDAN K., MURUGAN K., PANNEERSELVAM C., MAHESH KUMAR P., AMERASAN D., SUBRAMANIAM J., VINCENT S., BARNARD D.R., 2012b - Laboratory and field evaluation of medicinal plant extracts against filarial vector, Culex quinquefasciatus Say (Diptera: Culicidae). - Parasitol. Res. 110: 2105-2115.

MAHESH KUMAR P., MURUGAN K., KOVENDAN K., SUBRAMANIAM J., AMARESAN D., 2012 - Mosquito larvicidal and pupicidal efficacy of Solanum xanthocarpum (Family: Solanaceae) leaf extract and bacterial insecticide, Bacillus thuringiensis, against Culex quinquefasciatus Say (Diptera: Culicidae). - Parasitol. Res. 110: 2541-2550.

MAHESWARAN R., SATHIS S., IGNACIMUTHU S., 2008 - Larvicidal activity of Leucus aspera (Willd.) against the larvae of Culex quinquefasciatus Say. and Aedes aegypti. - Int. J. Int. Biol. 2: 214-217.

MANGATHAYARU K., THIRUMURUGAN D., PATEL P.S., PRATAP D.V., DAVID D.J., KARTHIKEYAN J., 2006 - Isolation and identification of nicotine from Leucas aspera (willd). - Ind. J. Pharm. Sci. 68: 88-90.

MISHRA A.K., CHAND S.K., BARIK T.K., DUA V.K., RAGHAVENDRA K., 2012 - Insecticide resistance status in Anopheles culicifacies in Madhya Pradesh, central India. - J. Vect. Borne. Dis. 49: 39-41.

PEERZADA N., 1997 - Chemical composition of the essential oil of Hyptis suaveolens. - Molecules. 2: 165-168.

PROMSIRI S., NAKSATHIT A., KRUATRACHUE M., THARAVA U., 2006 Evaluations of larvicidal activity of medicinal plant extracts to Aedes aegypti (Diptera: Culicidae) and other effects on a non target fish. - Insect. Sci. 13: 179-188. 
RAHUMAN A.A., GOPALAKRISHNAN G., VENKATESAN P., GEETHA K., BAGAVAN A., 2008 - Mosquito larvicidal activity of isolated compounds from the rhizome of Zingiber officinale. - Phytother. Res. 22: 1035-1103.

RUSSELL P.F., RAO T.R., 1942 - On the ecology of larvae of Anopheles culicifacies Giles, in borrow-pits. - Bull. Entomol. Res. 32: 341-361.

SABESAN S., KRISHNAMOORTHY K., JAMBULINGAM K., RAJENDRAN G., KUMAR P.N., RAJAGOPALAN P.K., 1986 - Breeding habitats of Anopheles culicifacies in Rameshwaram Island. - Ind. J. Med. Res. 84: 44-52.

SADHU S.K., OKUYAMA E., FUJIMOTO H., ISHIBASHI M., 2003 Separation of Leucas aspera, a medicinal plant of Bangladesh, guided by prostaglandin inhibitory and antioxidant activities. - Chem Pharm Bull (Tokyo). 51: 595-598.

SEYOUM A., PÅLSSON K., KUNG'A S., KABIRU E.W., LWANDE W., KILLEEN G.F., HASSANALI A., KNOTS B.G.J., 2002 - Traditional use of mosquitorepellent plants in western Kenya and their evaluation in semi field experimental huts against Anopheles gambiae: ethnobotanical studies and application by thermal expulsion and direct burning. - Trans. R. Soc. Trop. Med. Hyg. 96, 225-231.

SHARMA V.P., ANSARI M.A., 1994 - Personal protection from
Mosquitoes (Diptera: Culicidae) by burning neem oil in kerosene repellent action of neem. - J. Med. Entomol. 3: 95-102.

SHARMA S.K., DUA V.K., SHARMA V.P., 1995 - Field study on the mosquito repellent action of neem oil. - South Asian J. Trop. Med. Pub. Health. 26: 180-182.

SHARMA M., SAXENA R.C., 1996 - Sphaeranthus indicus as a mosquito larvicidae. - J. Appl. Zool. Res. 7: 87-88.

SINGH S.P., RAGAVENDHIRA K., SINGH R.K., SUBBARAO S.K., 2002 Studies on larvicidal prosperities of leaf extract of Solanum nigrum Linn (Family: Solanaceae). - Curr. Sci. 81: 1529.

SUBBARAO S.K., NANDA N., RAGHAVENDRA K., 1999 - Malariogenic stratification of India using Anopheles culicifacies sibling species prevalence. - ICMR Bulletin. 29: 75-80.

WHO., 1996 - Report of the WHO informal consultation on the evaluation on the testing of insecticides. CTD/WHO PES/IC/96.1. - World Health Organisation, Geneva, p 69.

WHO., 1998 - Test procedures for insecticide resistance monitoring in malaria vectors. Bio-efficacy and persistence of insecticides on treated surfaces. Report of the WHO informal consultation. Geneva WHO/CDC/MAL/ 98.12. - World Health Organisation, Geneva.

YOGANARASIMHAN S.W., 2000 - Medicinal plants of India. - Cyber Media, Bangalore, pp. 10-11. 